\title{
A Comparison of Objective and Subjective Measures of Respiration in Healthy Adults
}

\author{
Pranali Chavan'; Jyoti Mohite $^{2}$ \\ ${ }^{1}$ Assistant Professor at Audiology and Speech Therapy Department, B. Y. L. Nair Ch. Hospital \& Topiwala \\ National Medical College, Mumbai, Maharashtra, India. \\ ${ }^{2}$ Associate Professor at Audiology and Speech Therapy Department, B. Y. L. Nair Ch. Hospital \& Topiwala \\ National Medical College, Mumbai, Maharashtra, India.
}

Corresponding Author: Pranali Chavan

\begin{abstract}
Objective: The purpose of this study was to investigate the gender and age effect on lung function measures and maximum phonation time in the age range of 20-60 years. The present study also aimed to evaluate correlation between FEV1 and maximum phonation time.

Study Design: Cross- sectional study, Convenience sampling method

Method: The lung function measures (FVC, FEV1, \& FEV1/FVC) and maximum phonation time were obtained from 200 healthy participants in the age range of 20-60 years. These participants were divided into two groups (Group I - 20- 40 years \& Group II - 41- 60 years) with equal number of males and females in each group. The lung function measures were obtained using Easy on PC spirometer. Three consecutive trials were collected for MPT using stopwatch. Mann- Whitney U test was used to determine effect of age and gender on lung function measures and MPD. Spearman's Rank Correlations were performed to identify is there any significant relationships between the FEV1 and MPT of $/ \mathrm{a} /, / \mathrm{i} /, / \mathrm{u} /, / \mathrm{s} /$, and $/ \mathrm{z} /$.

Results: There were significant effect of gender on FVC, FEV1, \& MPT except on FEV1/FVC. There was significant effect of age in both genders on FVC, FEV1, \& FEV1/FVC. There was no significant effect of age in both genders on MPT /i/, /u/, /s/, and /z/ except MPT /a/ for males. FEV1 showed significant positive correlation with MPD $(/ \mathrm{a} /, / \mathrm{i} /, / \mathrm{u} /, / \mathrm{s} /$, and $/ \mathrm{z} /)$.

Conclusions: The mean FVC and FEV1 were lower in females and higher in males in both the age groups. Maximum phonation time of $/ \mathrm{a} /, / \mathrm{i} /, / \mathrm{u} /, / \mathrm{s} /$, and $/ \mathrm{z} / \mathrm{l}$ was longer in males as compared to female. Age has inverse effect on lung function measure and MPT. FEV1 showed positive correlation with MPT, which indicates that FEV1 increases with increase in MPT.
\end{abstract}

Key Words: Maximum Phonation Time, Lung Function Measures

\section{INTRODUCTION}

The production of voice depends on the interaction between pulmonary aerodynamic forces and the laryngeal myoelastic forces. Respiration is not only important for voice production but also can serve as an indicator of voice disorder. In cases with voice disorder when any mass lesion, poor muscle coordination, or aging affects mucosal covering and muscles of vocal fold it causes incomplete glottal closure, increased airflow rate, and decreased supraglottic air pressure. ${ }^{[1]}$ Therefore an SLP needs to include respiratory evaluation as a routine clinical procedure.

In cases with voice disorder mass lesion or poor muscle coordination affects mucosal covering and muscles of vocal fold leading to incomplete glottal closure, increased airflow rate, and decreased supraglottic air pressure. ${ }^{[1]}$

Spirometry is a widely used routine objective examination of pulmonary 
function and its disorders. ${ }^{[2]}$ The 3 crucial lung function measures which help us to diagnose respiratory disorders are Forced vital capacity (FVC), Forced expiratory volume- one second (FEV1), and FEV1/ FVC ratio. ${ }^{[3]}$

\section{Explanation of common test values in pulmonary function tests}

\begin{tabular}{|l|l|}
\hline Test & Description \\
\hline Forced Vital Capacity & $\begin{array}{l}\text { It is a measurement of the lung volume (in litres) represented by the volume of air in the lungs that can } \\
\text { be maximally exhaled following a deep inhalation. }{ }^{[4]}\end{array}$ \\
\hline $\begin{array}{l}\text { Forced expiratory volume }- \\
\text { one Second (FEV1) }\end{array}$ & $\begin{array}{l}\text { It is a measure of volume of air that can be exhaled in one second following a deep inhalation. It is } \\
\text { measured in liters. }{ }^{[4]}\end{array}$ \\
\hline FEV1/ FVC & $\begin{array}{l}\text { It is the ratio of FEV1 to FVC. In a healthy adult, FVC and FEV1 values lies between } 80 \% \text { to } 120 \% \text { and } \\
\text { FEV1/ FVC is within 5\% of the predicted value. }{ }^{[4]}\end{array}$ \\
\hline
\end{tabular}

It is well known that lung function measures vary across the life span. In healthy individuals, pulmonary function is affected by age, gender, height, ethnicity, genetic, environmental, socio-economic status, and some other unidentified factors. ${ }^{[5-7]}$ The FVC and FEV1 decrease as a function of age. ${ }^{[8-10]}$ In various studies, it was reported that males have a higher value of pulmonary measures than females. ${ }^{[11,12]}$ Singh et al. ${ }^{[13]}$ studied spirometric measures on 1,999 subjects (1,385 males and 614 females). They reported mean forced vital capacity (FVC) in males was 3.4910 liters and in females, it was 2.510 liters. In the same ethnic and age groups, variations in vital capacity were noted in relation to their height in males. ${ }^{[14]}$ Kamat et al. ${ }^{[15]}$ and Udwadia et al. ${ }^{[16]}$ reported that Indians have lower VC than westerners. Also lung capacity is dependent more upon height and weight than race.

Instruments needed for assessment are not available in every setup, as they are expensive. Therefore clinicians have to depend on non-instrumental assessment which has been a boon for the clinicians. Maximum phonation time (MPT) and s/z ratio are two such subjective methods useful to assess respiratory and phonatory efficacy. ${ }^{[17,18]}$ Maximum phonation time and $\mathrm{s} / \mathrm{z}$ ratio are measures of phonation time. ${ }^{[17]}$ Maximum phonation time is the maximum length of time that a patient can sustain phonation on a single breath. Most normal adults can sustain vowel production for 1520 seconds. $^{[19]}$ However, age and genderwise differences in MPT have been reported with males showing higher MPT as compared to females. And in both males and females MPT declines with age. ${ }^{[20-22]}$ Reduced MPT is suggestive of insufficient respiratory functioning. In clients with voice disorders, MPT is reduced. ${ }^{[23]}$

According to Yanagihara et al. ${ }^{[24]}$ phonation volume bears a linear relationship with VC. Lima et al. ${ }^{[1]}$ studied the correlation between slow vital capacity and maximum phonation time in 101 healthy adults. They concluded that there was a positive correlation between slow vital capacity and maximum phonation time of /a/, /s/, and /z/in healthy adult subject. Similarly, MPT showed positive correlations to the FVC and FEV1 in subjects with and without dysphonia. ${ }^{[25]}$

Adequate breath support is a prerequisite for phonation. Hence, assessment of breathing should ideally be a part of routine SLP practice. Therefore, it is important to find out the correlation between maximum phonation duration and lung measures. Hence the present research aimed to study the gender and age effect on lung function measures and maximum phonation time in individuals in the age range of $20-60$ years. Secondly, the relationship between lung function and MPT was also studied.

\section{MATERIAL AND METHOD 2.1 Participants}

A total of 200 participants (100 males \& 100 females) in the age range of 20- 60 years were recruited after obtaining approval from the ECARP committee. Both the genders were divided evenly into two age groups (Group I - 20-40 years \& Group 
II 41-60 years). In Group I, there were 50 males with a mean age of $28.24 \pm 5.68$ and 50 females with a mean age of $24.72 \pm 4.51$. Similarly in Group II, there were 50 males with a mean age of $49.6 \pm 5.70$ and 50 females with a mean age of $47.52 \pm 5.29$. Participants were recruited from the hospital. Medical and nonmedical staff, students, other relatives, and caregivers of patients coming for therapy, fulfilling the inclusion criteria was taken up for the study. All participants with no respiratory illness and normal voice quality (GRBAS < 1) were included in the study. s/z ratio for all participants was <1.0. All participants selfreported normal vocal functioning. Participants with a past/present history of voice disorder, smoking, lung disease, heart disease, frequent URTI, and pregnant women were excluded from the study.

\subsection{Spirometry procedure:}

A lung function test was carried out using an Easy on - PC spirometer. This spirometer meets ATS/ERS (2005) specification. It provides real-time graphs and an animated incentives screen ${ }^{[4]}$. A disposable mouthpiece coupled to the spirometer was used to avoid contamination. The test procedure was explained and a demonstration of the manoeuvres was given. Activities like eating a heavy meal; smoking and vigorous exercises before testing were avoided. Participants were instructed to avoid tight clothing on the day of testing. If clothes are too tight it may give restrictive pictures on spirometry. 1 The test was carried out with the subject sitting in the upright position on the chair. A nose clip was used to avoid leakage of the air.

The subjects were asked to hold the mouthpiece lightly with the help of teeth. Lips were tightly closed around the mouthpiece to obtain a good seal. Then participants were instructed to breathe normally a couple of times. After a few breaths, the subject was instructed to quickly take a deep and full breath inside. Forcefully blow out air for a minimum of 68 seconds and again take a deep breath inward. Repeated verbal prompts were given to encourage the participants to sustain the exhalation for at least $6 \mathrm{sec}$.

Based on a review of literature forced vital capacity (FVC), forced expiratory volume in one second (FEV1) and the ratio of FEV1/FVC was found to be the most widely used parameters of spirometry for differential diagnosis of respiratory disorders. Hence, FVC, FEV1, and FEV1/FVC parameters were included in the study. Observed maximum values of FVC and FEV1 were noted and compared with predicted values. To compute the predicted values, it is necessary to enter the sex, age, height, weight, and ethnic group of the subject. The percent predict is calculated by dividing the pre-best value (value obtain from the best trial) by the predicted value and multiplying by 100 . The ratio of FEV1/ FVC was compared with the predicted ratio. ${ }^{[4]}$ Standardized criteria for reproducibility of the test (the difference between the two largest values should be $>150 \mathrm{~mL}$ )was used. ${ }^{[26]}$

\subsection{Maximum phonation time procedure:}

The participant was seated in an upright posture and instructed to take a deep breath to the extent possible and while exhaling phonate /a/ sound for as long as possible. Using a digital stopwatch time was noted. When the subject couldn't follow the instruction, a demonstration of MPT was given. 3 trials were given and the highest maximum phonation time of 3 trials was considered for the study. The rest period of 30 seconds was given between 3 trials. Similarly MPT for /i/, /u/, /s/, and /z/ was elicited.

\section{STATISTICAL ANALYSIS}

Statistical analysis was done using SPSS 25.0 software. ${ }^{[27]}$ The descriptive data in terms of mean and standard deviation was documented. Comparison between gender and two age groups ( 20 to $40 \& 41$ to 60 ) was done using the Mann-Whitney U test as all the data failed the 'Normality' test. Correlation between FEV1 and MPT was done by using Spearman's rank correlation. 


\section{RESULTS}

The mean and standard deviation for FVC, FEV1, FEV1/FVC, MPT /a/, /i/, /u/, $/ \mathrm{s} /$, and $/ \mathrm{z} /$ are provided in Table 1 . The habitual breathing pattern of the participants was noted. $1 \%$ participants showed clavicular breathing, $36 \%$ participants showed thoracic breathing, 25\% participants showed thoracic-abdominal breathing. Abdominal breathing pattern was observed in most of the cases, $38 \%$ of participants showed abdominal breathing pattern.

\subsection{Effect of gender on lung function measures and MPT}

Table 1. Comparison of the MPT /a/, MPT /i/, MPT /u/, MPT /s/, MPT /z/, FVC, FEV1 between males and females

\begin{tabular}{|c|c|c|c|c|c|}
\hline \multirow[b]{3}{*}{ Variables } & \multicolumn{4}{|c|}{ Gender } & \multirow[b]{3}{*}{$P$ value } \\
\hline & \multicolumn{2}{|c|}{ Female } & \multicolumn{2}{|l|}{ Male } & \\
\hline & Mean & SD & Mean & SD & \\
\hline MPT /a/ & 14.25 & 3.73 & 16.79 & 4.99 & $<0.001^{*}$ \\
\hline MPT /i/ & 16.35 & 4.62 & 18.62 & 6.25 & $0.008^{*}$ \\
\hline MPT /u/ & 15.15 & 4.43 & 17.11 & 5.52 & $0.003 *$ \\
\hline MPT /s/ & 9.81 & 4.36 & 10.79 & 3.84 & $0.012 *$ \\
\hline MPT /z/ & 12.61 & 4.42 & 13.89 & 4.60 & $0.019 *$ \\
\hline FVC & 2.76 & 0.45 & 3.44 & 0.72 & $<0.001^{*}$ \\
\hline FEV1 & 2.33 & 0.42 & 2.89 & 0.64 & $<0.001 *$ \\
\hline FEV1/FVC & 0.84 & 0.05 & 0.84 & 0.04 & 0.245 \\
\hline
\end{tabular}

The Mann - Whitney U test result revealed that FVC, FEV1, MPT /a/, /i/, /u/, $/ \mathrm{s} /$, and $/ \mathrm{z} / \mathrm{were}$ significantly higher in males than that in females $(p<0.05$, Table
1). However, no significant difference was observed for FEV1/ FVC ratio in males and females $(p=0.245)$. The effect size was small for MPT of /i/, /u/, /s/, and/z/ except for /a/, it was medium. For FVC and FEV1 large effect size was noted.

\subsection{Effect of age on lung function measures and MPT}

As the gender effect was significant, the effect of age was done separately in males and females. Mann - Whitney U test was used to analyze the effect of age on phonation time and lung measures in males and females.

\subsubsection{Effect of age on lung function measures and MPT in females}

The Mann- Whitney U test results revealed that FVC, FEV1, and FEV1/FVC were significantly higher in group I when compared with group II participants $(p<$ 0.05 , Table 2). However, MPT /a/ $(\mathrm{p}=$ $0.150)$, MPT $/ \mathrm{i} /(\mathrm{p}=0.934)$, MPT $/ \mathrm{u} /(\mathrm{p}=$ $0.646), \mathrm{MPT} / \mathrm{s} /(\mathrm{p}=0.759)$, and MPT /z/ $(\mathrm{p}=0.444)$ did not show any significant age effect. The effect size was large for FVC and FEV1 but for FEV1/FVC ratio, it was medium.

Table 2. Comparison of the MPT /a/, MPT /i/, MPT /u/, MPT /s/, MPT /z/, FVC, FEV1 and FEV1/FVC between group I and group II in males and females.

\begin{tabular}{|c|c|c|c|c|c|c|c|c|c|c|}
\hline \multirow[b]{3}{*}{ Variables } & \multicolumn{4}{|l|}{ Males } & \multirow[b]{3}{*}{$P$ value } & \multicolumn{4}{|c|}{ Female } & \multirow[b]{3}{*}{$P$ value } \\
\hline & \multicolumn{2}{|c|}{ 20- 40 years } & \multicolumn{2}{|c|}{ 41- 60 years } & & \multicolumn{2}{|c|}{ 20- 40 years } & \multicolumn{2}{|c|}{ 41- 60 years } & \\
\hline & Mean & SD & Mean & SD & & Mean & SD & Mean & SD & \\
\hline MPT /a/ & 17.79 & 4.91 & 15.77 & 4.90 & $0.015^{*}$ & 17.79 & 4.91 & 15.77 & 4.90 & $0.015^{*}$ \\
\hline MPT /i/ & 19.59 & 6.22 & 17.64 & 6.18 & 0.064 & 19.59 & 6.22 & 17.64 & 6.18 & 0.064 \\
\hline MPT /u/ & 17.82 & 5.27 & 16.34 & 5.69 & 0.066 & 17.82 & 5.27 & 16.34 & 5.69 & 0.066 \\
\hline MPT /s/ & 10.91 & 3.24 & 10.66 & 4.38 & 0.707 & 10.11 & 4.76 & 9.51 & 3.94 & 0.759 \\
\hline MPT /z/ & 14.22 & 4.16 & 13.55 & 5.02 & 0.296 & 12.92 & 4.40 & 12.29 & 4.46 & 0.444 \\
\hline FVC & 3.70 & 0.72 & 3.16 & 0.60 & $0.0001 *$ & 3.70 & 0.72 & 3.16 & 0.60 & $0.0001 *$ \\
\hline FEV1 & 3.14 & 0.65 & 2.63 & 0.52 & $<0.001^{*}$ & 3.14 & 0.65 & 2.63 & 0.52 & $<0.001^{*}$ \\
\hline FEV1/FVC & 0.84 & 0.04 & 0.82 & 0.04 & $0.051 *$ & 0.84 & 0.04 & 0.82 & 0.04 & $0.051 *$ \\
\hline
\end{tabular}

\subsubsection{Effect of age on lung function measures and MPT in males}

The Mann- Whitney U test results revealed that FVC, FEV1, FEV1/FVC, and MPT /a/ were significantly higher in group I when compared with group II participants $(p$ $<0.05$, Table 2). However, MPT $/ \mathrm{i} /(\mathrm{p}=$ $0.064)$, MPT $/ \mathrm{u} /(\mathrm{p}=0.066)$, MPT $/ \mathrm{s} /(\mathrm{p}=$
0.070), and MPT /z/ $(\mathrm{p}=0.296)$ did not show any significant age effect. The effect size was large for FVC and FEV1 except for FEV1/FVC ratio, it was medium. For MPT of /a/ effect size was small. 


\subsection{Correlation between maximum phonation duration $(/ \mathrm{a} /, \mathrm{i} /, / \mathrm{u} /, / \mathrm{s} /, / \mathrm{z} /)$ in seconds and FEV1in litres}

Spearman's rank order correlation coefficient rho was calculated at 0.01 levels (2 tailed). FEV1 showed significant correlation with all MPT measures $[/ \mathrm{a} / \mathrm{r}=$ $0.359, \mathrm{p}=1.00 \mathrm{E}-06), \quad / \mathrm{i} / \quad \mathrm{r}=0.265, \mathrm{p}=$ $0.0002), / \mathrm{u} /(\mathrm{r}=0.302, \mathrm{p}=1.39 \mathrm{E}-05), / \mathrm{s} /(\mathrm{r}=$ $0.262, \mathrm{p}=0.0002)$ and $/ \mathrm{z} /(\mathrm{r}=0.270, \mathrm{p}=$ 0.0001)]. A positive correlation was observed with all the measures. This indicates that MPT increases with an increase in FEV1. (Table 3)

Table 3: Correlation between maximum phonation time and FEV1

\begin{tabular}{|c|c|c|}
\hline Variables & Spearman's rho & FEV1 (Liters) \\
\hline \multirow[t]{2}{*}{ MPT/a/ (Sec) } & Correlation Coefficient & .359 \\
\hline & $\mathrm{P}$-value & $<0.0001 *$ \\
\hline \multirow[t]{2}{*}{$\mathrm{MPT} / \mathrm{u} /(\mathrm{Sec})$} & Correlation Coefficient & .302 \\
\hline & P-value & $<0.0001 *$ \\
\hline \multirow[t]{2}{*}{ MPT /i/ (Sec) } & Correlation Coefficient & .265 \\
\hline & $\mathrm{P}$-value & $0.0002 *$ \\
\hline \multirow{2}{*}{ MPT /s/ (Sec) } & Correlation Coefficient & .262 \\
\hline & $\mathrm{P}$-value & $0.0002^{*}$ \\
\hline \multirow{2}{*}{ MPT /z/ (Sec) } & Correlation Coefficient & .270 \\
\hline & $\mathrm{P}$-value & $0.0001 *$ \\
\hline
\end{tabular}

\section{DISCUSSION}

Voice production mainly depends upon the respiratory system. As respiration plays a major role in voice production, it is important to assess respiration during the assessment of the voice.FVC, FEV1, and FEV1/ FVC are 3 important measures of lung function. The present study aimed to evaluate a relationship of lung function measures as measured by FVC and FEV1 with the maximum phonation time of /a/, /i/, $/ \mathrm{u} /, / \mathrm{s} /$, and $/ \mathrm{z} /$ in the age range of $20-60$ years.

In the present study the effect of gender was observed on lung measures, Males showed higher FVC and FEV1 values than females, which can be attributed to inherent anatomical variations in the two genders. However, FEV1/ FVC ratio was not significantly different between the genders. Zakaria et al. ${ }^{[11]}$ reported in their study that males showed a higher value of pulmonary function measures than in females, but not for the FEV1/FVC ratio. In contrast, Banerjee et al. ${ }^{[12]}$ reported that there was a significant difference between males and females for FEV1 and FEV1/ FVC, but not for FVC value. The differences in the findings could be attributed to the methodological differences. Smokers were included in their study. Also, subjects studied were non-asthmatic having some other pulmonary issues. The significant age effect of age was also noted for MPT of $/ \mathrm{a} /, / \mathrm{i} /, / \mathrm{u} /, / \mathrm{s} /$, and /z/. These findings are in agreement with previous studies done by Hirano et al., ${ }^{[28]}$ Maslan et al., ${ }^{[29]}$ Shanks et al. ${ }^{[30]}$

The present study also found that there was no significant difference between the two age groups for all measures of MPT, except for vowel /a/ where two groups differed significantly in males. Knuijt et al. ${ }^{[31]}$ reported MPT of /a/ was significantly different in males between two age groups, probably because it was a first measure obtained where participants could give their best performance. These findings contradict the findings reported by Awan et al. ${ }^{[32]}$ where they reported that age showed a significant correlation with maximum phonation time.

The lung function measure obtained in females between group I and group II were compared. The result revealed that there was a statistically significant difference between the two age groups. Similar findings were obtained in males between two age groups with the older age group performing poorer than the younger age group. Thus, it can be concluded that as age increases lung function decrease in both males and females, probably due to changes in elasticity of the lung tissue as a part of normal aging. The present study results are in accordance with findings by VazFragoso et al. ${ }^{[8]}$ where they reported that the FEV1 value decreases progressively in middleaged and older persons. This decrease in FEV1 may be caused due to normal aging and the onset and progression of cardiopulmonary disease.

The results of the present study are also in accordance with the study done 
by Stanojevic et al. ${ }^{[9]}$ where they found that there was a decline in FEV1/ FVC ratio as age increases. Similar findings were also reported by Behera et al., ${ }^{[10]}$ where they found that FEV1 and FEV1/ FVC ratio decreases as age increases.

It was found that there was a correlation between the MPT of $/ \mathrm{a} /, / \mathrm{i} /, / \mathrm{u} /$, and FEV1. FEV1 shows a statistically significant positive correlation with MPT of /a/, /i/, and /u/. This shows as FEV1 increases MPT also increases. This is probably due to the nature of the tasks. As both the tasks (MPT and FEV1) require the air to be sustained. The findings of the present study are in accordance with the study done by Hassan et al. ${ }^{[25]}$ where they reported that there was a positive correlation between maximum phonation time and pulmonary function measures. The contrast findings were noted in the study by Cassiani et al. ${ }^{[3]}$ They found that there was no correlation between FEV1 and MPD in control as well as in the study group. These contrasting findings may be due to different age groups and the number of participants studied between the two studies.

\section{CONCLUSION}

To conclude, maximum phonation time and lung measures gets affected by gender. Males have higher lung capacities than females. Also, males have a higher maximum phonation time as compared to females. Age has an inverse correlation with phonation time and lung measures. This study also concluded that there was a positive correlation between FEV1 and MPT.

Acknowledgement: None

Conflict of Interest: None

Source of Funding: None

Ethical Approval: Approved

\section{REFERENCES}

1. Lima DCB de, Palmeira AC, Costa EC, Mesquita FO de S, Andrade FMD de, Correia Júnior MA de V. Correlation between slow vital capacity and the maximum phonation time in healthy adults. Rev CEFAC 2014;16(2):592-7.

2. Pellegrino R, Viegi G, Brusasco V, Crapo RO, Burgos F, Casaburi R, et al. Interpretative strategies for lung function tests. Eur Respir J 2005;26(5):948-68.

3. Moore VC. Spirometry: Step by step. Breathe 2012;8(3):233-40.

4. Barreiro T, Perillo I. An Approach to Interpreting Spirometry - American Family Physician. 2004.

5. Chhabra S k, Kumar R, Gupta U, Rahman M, Dash DJ. Prediction equations for spirometry in adults from northern India. Indian J Chest Allied Sci 2014;56(4):221-9.

6. Dikshit MB, Raje S, Agrawal MJ. Lung functions with spirometry: An Indian perspective-I. Peak expiratory flow rates. Indian J. Physiol. Pharmacol.2005;49(1):818.

7. Amit Bandyopadhay, Ishita Bhattacharjee, Rishna Dalui SP. Pulmonary Function Studies of Healthy Non-smoking Male University Students of Kolkata, India Revisited. Malays J Med Sci 2013;20(2): 17-24.

8. Vaz Fragoso CA, McAvay G, Van Ness PH, Metter EJ, Ferrucci L, Yaggi HK, et al. Aging-related considerations when evaluating the forced expiratory volume in 1 second (FEV1) over time. J Gerontol - Ser Biol Sci Med Sci 2016;71(7):929-34.

9. Stanojevic S, Wade A, Stocks J, Hankinson $\mathrm{J}$, Coates AL, Pan H, et al. Reference ranges for spirometry across all ages: A new approach. Am J Respir Crit Care Med 2008; 177(3):253-60.

10. Behera AA, Behera BK, Dash $\mathrm{S}$ et. al. Variation of pulmonary function tests with relation to increasing age in healthy adults. Int J Health Sci Res. 2014;4(3):136-141.

11. Zakaria R, Harif N, Al-Rahbi B, Aziz CBA, Ahmad AH. Gender differences and obesity influence on pulmonary function parameters. Oman Med J 2019;34(1):44-8.

12. Banerjee J, Roy A, Singhamahapatra A, Dey PK, Ghosal A, Das A. Association of body mass index with lung function parameters in non-asthmatics identified by 
spirometric protocols. J Clin Diagn Res 2014;8(2):12-4.

13. Singh R, Singh HJ, Sirisinghe RG. Spirometric studies in Malaysians between 13 and 69 years of age. Med J Malays 1993;48(2): 175-84.

14. Urooj Bhatti, Keenjher Rani MQM. Variation in lung volumes and capacities among young males in relation to height. $\mathrm{J}$ Ayub Coll Abbottabad 2014;26(2):200-2.

15. Kamat, Tyagi NK, Rashid SSA. Lung Function In Indian Adult Subjects. Lung India 1982;1(1):21.

16. Udwadia FE, Sunavala JD, Shetye VM, Jain PK. The maximal expiratory flow-volume curve in normal subjects in India. Chest 1986;89(6):852-6.

17. Colton RH, Casper JK, Leonard RJ. Understanding voice problem: A physiological perspective for diagnosis and treatment: Fourth edition. Wolters Kluwer Health Adis (ESP), 2011. 494 p.

18. GS Neiman BE. Procedural aspects of eliciting maximum phonation time. Folia Phoniatr Basel 1981;33(5):285-93.

19. Prater, Rax J RWS. Manual of Voice Therapy. Austin: Tex: PRO-ED; 1984.

20. Lewis K, Casteel R, McMahon J. Duration of Sustained /a/ Related to the Number of Trials. Folia Phoniatr Logop 1982;34(1):418.

21. Hirano M. Clinical examination of voice. Wein: Springer-Verlag; 1981.

22. Kreul EJ. Neuromuscular control examination (NMC) for Parkinsonism: vowel prolongations and diadochokinetic and reading rates. $J$ Speech Hear Res 1972;15(1):72-83.

23. Speyer R. Effects of Voice Therapy: A Systematic Review. J. Voice. 2008; 22(5): 565-80.

24. Yanagihara N, von Leden $\mathrm{H}$. Respiration and Phonation. Folia Phoniatr Logop 1967; 19(3):153-66.
25. Hassan MM, Hussein MT, Emam AM, Rashad UM, Rezk I, Awad AH. Is insufficient pulmonary air support the cause of dysphonia in chronic obstructive pulmonary disease? Auris Nasus Larynx 2018;45(4):807-14.

26. Miller MR, Hankinson J, Brusasco V, Burgos F, Casaburi R, Coates A, et al. Standardisation of spirometry. Eur. Respir. J.2005;26(2):319-38.

27. Corp. I. IBM SPSS Statistics for Windows. 2017;

28. Hirano $M$, Koike $Y$, von Leden $H$. Maximum Phonation Time and Air Usage During Phonation. Folia Phoniatr Logop 1968;20(4):185-201.

29. Maslan J, Leng X, Rees C, Blalock D, Butler SG. Maximum phonation time in healthy older adults. J Voice 2011;25(6): 709-13.

30. Shanks SJ, Mast D. Maximum duration of phonation: objective tool for assessment of voice. Percept Mot Skills 1977;45(3 II):1315-22.

31. Knuijt S, Kalf J, Van Engelen B, Geurts A, de Swart B. Reference values of maximum performance tests of speech production. Int J Speech Lang Pathol 2019;21(1):56-64.

32. Awan SN. The aging female voice: Acoustic and respiratory data. Clin Linguist Phon 2006;20(2-3):171-80.

33. Cassiani R de A, Aguiar-Ricz L, Santos CM dos, Martinez JAB, Dantas RO. Glottal competence in chronic obstructive pulmonary disease. Audiol - Commun Res 2013;18(3):149-54.

How to cite this article: Chavan P, Mohite J. A comparison of objective and subjective measures of respiration in healthy adults. Int $J$ Health Sci Res. 2021; 11(10): 60-66. DOI: https://doi.org/10.52403/ijhsr.20211009 